\title{
Calidad de vida, aspectos psicológicos y sociales en pacientes con infección VIH avanzada
}

\author{
I. MARTÍN SUÁREZ, R. CANO MONCHUL, P. PÉREZ DE AYALA, M. AGUAYO \\ CANELA, F. CUESTA, P. RODRÍGUEZ, E. PUJOL DE LA LLAVE
}

Servicio de Medicina Interna. Hospital Juan Ramón Jiménez. Huelva

\author{
QUALITY OF LIFE, PSYCHOLOGICAL AND SOCIAL ASPECTS IN \\ PATIENTS WITH ADVANCED HIV DISEASE
}

\begin{abstract}
RESUMEN
Fundamento: La infección VIH avanzada conlleva un importante deterioro físico, psíquico y de calidad de vida para el paciente. La introducción de nuevas estrategias de tratamiento probablemente impliquen un beneficio global para los pacientes. Se realiza este estudio con el objetivo de estudiar en pacientes VIH avanzados; la situación clínica, la prevalencia de alteraciones psicológicas(ansiedad y depresión), la calidad de vida y el impacto sobre estas variables de la introducción de nuevos fármacos antirretrovirales.

Métodos: Estudio observacional prospectivo realizado entre enero de 1996 y junio de 1997 con 52 pacientes VIH avanzados (recuentos CD4 menores de $200 \mathrm{cel} / \mathrm{\mu l}$ ). Se realiza 2 entrevistas clínicas con un intervalo de 12 meses introduciéndose tras la primera un cambio de estrategia de tratamiento antirretroviral. Para la valoración la calidad de vida se utiliza el MOS SF-36. Para la valoración de la ansiedad y depresión se utilizaron el STAI y la escala de Beck respectivamente.

Resultados: Existe una importante deterioro físico al inicio del estudio con un $84,6 \%$ de pacientes polisintomáticos. Encontramos una elevada prevalencia de ansiedad $(76,9 \%)$ y depresión $(86,6 \%)$ que alcanza criterios de gravedad en el $75 \%$ y $25 \%$ de los casos respectivamente. La calidad de vida se ve comprometida severamente con afectación de la mayoría de las dimensiones del SF-36 conservándose la función social. La introducción del cambio de estrategia terapéutica produce una reducción de polisintomáticos a $54,8 \%(\mathrm{p}=0,05)$, de la gravedad de la ansiedad $(\mathrm{p}=0,009)$ y depresión $(\mathrm{p}=0,05)$ y mejoría en la percepción de salud general $(\mathrm{p}=0,03)$ y alteraciones de rol físico $(\mathrm{p}=0,02)$.

Conclusiones: La infección VIH avanzada conlleva una alta carga sintomática con una elevada prevalencia de trastornos de la esfera psicológica y deterioro de la calidad de vida. Las nuevas estrategias de tratamiento antirretroviral inducen una mejoría global a los pacientes, pero hace necesario un abordaje de los trastornos psicológicos.
\end{abstract}

PALABRAS CLAVE: Infección VIH. Enfermedad avanzada. Calidad de vida.

\begin{abstract}
Objective: The advanced HIV infection carries an important physi cal, psychological and quality of life deterioration for patients. The introduction of new treatment strategies probably implies a global bene fit for HIV patients. We carried out this study in order to know in HIV advanced patients; the clinical situation, the prevalence of psychological alterations (anxiety and depression), the quality of life and the impact of the introduction of new antiretroviral drugs about these variables.

Methods: This is a prospective observational study carried out bet ween January of 1996 and June of 1997 with 52 advanced HIV patients (CD4 cells less than $200 / \mu l)$. We carried out 2 clinical interviews with an interval of 12 months being introduced after the first one a change of strategy of antiretroviral treatment. For evaluating the quality of life, the MOS SF-36 was used. The valuation of the anxiety and depression were carried out with the instruments STAI and Beck respectively.

Results: An important physical deterioration was found at the begin ning of the study with $84.6 \%$ of polisymptomatic patients. We found a high prevalence of anxiety (76.9\%) and depression (86.6\%) that reaches criteria of graveness respectively in $75 \%$ and $25 \%$ of the cases. The qua lity of life was severely affected involving most of the dimensions of the $S F-36$ scale preserving the social function. The introduction of the chan ge of therapeutic strategy produces a reduction of polisymptomtics patients to $54.8 \%(p=0.05)$, of the graveness of the anxiety $(p=0.009)$ and depression $(p=0.05)$ and improving in the perception of general health $(p=0.03)$ and alterations of physical role $(p=0.02)$.

Conclusions: The advanced HIV infection carries a high symptoma tic load with a high prevalence of psychological dysfunctions and dete rioration of the quality of life. The new strategies of HIV treatment indu ce a global improvement to the patients, but it makes necessary a better management of the psychological dysfunctions.
\end{abstract}

KEY WORDS: HIV infection. Advanced disease. Quality of life.

Martín Suárez I, Cano Monchul R, Pérez de Ayala P, Aguayo Canela M, Cuesta F, Rodríguez P, Pujol de la Llave E. Calidad de vida, aspectos psicológicos y sociales en pacientes con infección VIH avanzada. An Med Interna (Madrid) 2002; 19: 396-404.

\section{INTRODUCCIÓN}

A lo largo de estos últimos 15 años de pandemia por infección VIH, se han logrado grandes avances terapéuticos. Se dispone de fármacos antirretrovirales cada vez más potentes y eficaces, aunque incapaces de eliminar el virus del organismo, de elevado costo y no exentos de efectos secundarios. Asimismo, se ha conseguido un mejor manejo de las complicaciones relacionadas con la infección. Este hecho junto con la aparición de resistencias a las diferentes

Trabajo aceptado: 22 de mayo de 2002

Correspondencia: Ignacio Martín Suárez. C/ Puerto nº $143^{\circ}$ B. 21003 Huelva. Tel.: 95925 43 79. e-mail: imsuarez@ navegalia.com. 
estrategias farmacológicas ha dado como resultado un aumento de la supervivencia de los pacientes incluso en las etapas más avanzadas de la enfermedad. En este período toman preponderancia aspectos como la prevención y el manejo integral del paciente en el que se valoran aspectos nutricionales, de la esfera psicológica, social y de calidad de vida. La introducción en el mercado de nuevos fármacos antirretrovirales y con estrategias que tienden a la terapia precoz y combinada ha supuesto un cambio de mentalidad en el manejo de la infección VIH. Se persigue un mayor y más duradero control de la replicación viral intentando evitar la aparición de resistencias hecho que ya se ha puesto de manifiesto en algunos estudios. Sin embargo con las estrategias previas, la limitada eficacia del uso de antirretrovirales en situación de enfermedad avanzada quedaba eclipsada por los efectos secundarios y se llegaba a empeorar de manera global la calidad de vida de algunos pacientes (1-3) replanteándose en algunos casos el uso de antirretrovirales en estadios finales de la enfermedad (4-6). La crisis multidimensional que el SIDA ha provocado en la sociedad junto con el importante impacto sobre la condición física de quien lo padece favorece la aparición de respuestas de ansiedad y depresivas de forma sistemática (7). El término de calidad de vida representa el efecto funcional de una enfermedad y su terapéutica consecuente tal como es percibida por el individuo (8) y cuya influencia se extiende en tres ámbitos: la función física, función social y emocional (9). Se ha puesto de manifiesto una repercusión negativa sobre la calidad de vida en el paciente con infección VIH, mayor cuanto más avanzada está la enfermedad (10-14). Además, existe una clara relación entre el bienestar psicológico y la salud percibida por el paciente (15-17), presentando también un importante efecto negativo otras variables como el estado nutricional, la adicción a drogas vía parenteral como práctica de riesgo, sexo femenino, la accesibilidad a los sistemas sanitarios y el status socioeconómico (18-23).

Nos planteamos como objetivos de este trabajo, el estudio en pacientes con infección VIH avanzada de la prevalencia de alteraciones de la esfera psicológica (ansiedad y depresión), la valoración del estado de salud percibido(calidad de vida) en sus diferentes dimensiones y evaluar el impacto que ha tenido la introducción de estrategias terapéuticas de tratamiento antirretroviral con nuevos análogos de nucleósidos e inhibidores de la proteasa, en la situación clínica, inmunológica, psicológica y del estado de salud percibido.

\section{MATERIAL Y MÉTODOS}

\section{PACIENTES}

El estudio ha sido desarrollado entre Enero de 1996 y Junio de 1997 en el Servicio de Medicina Interna del Hospital Juan Ramón Jiménez de Huelva utilizando como población a estudio a los pacientes con infección VIH que vienen siguiéndose regularmente en la consulta externa de Enfermedades Infecciosas de este Hospital. La población diana la forman los pacientes con infección VIH demostrada, en situación de inmunodepresión avanzada (catalogables dentro del grupo 3 del CDC de 1993 al tener recuentos de CD4 menores de 200 células/ $\mu \mathrm{l}$.). La muestra es constituida no probabilísticamente a partir de todos los pacientes que cumplieran los criterios de inclusión a inicios de 1996 y aceptaran mediante consentimiento informado participar en el estudio. Un total de 52 pacientes fueron incluidos en el estudio. Se realizaron 2 entrevistas clínicas por cada paciente una al inicio del estudio y la segunda aproximadamente a los 12 meses. La entrevista clínica fue llevada a cabo por un médico internista familiarizado con el manejo de pacientes con infección VIH y un psicólogo familiarizado con el uso de los instrumentos que fueron empleados para el análisis de la calidad de vida y las variables de la esfera psicológica. Tras la primera entrevista, los pacientes fueron sometidos a un cambio en la estrategia de tratamiento antirretroviral que se llevó a cabo según la situación de cada paciente y siguiendo las recomendaciones internacionales en el momento del estudio (24-27). Se realizó una recogida sistemática y protocolizada de toda la actuación clínica y de múltiples datos extraídos de ella, con registro en un soporte informatizado.

\section{VARIABLES EPIDEMIOLÓGICAS, CLÍNICAS Y ANALÍTICAS}

Se analizaron variables epidemiológicas como situación familiar personal y social. Para la medición de la clase social y cultural se utilizaron los indicadores aconsejados por el grupo de trabajo de la Sociedad Española de Epidemiología: Clasificación de ocupaciones de 1994 (CNO-94), nivel de estudios según una escala empleada por el Departamento de estadística de la Comunidad de Madrid y el nivel anual de ingresos brutos. Se analizaron además antecedentes penales, hábitos tóxicos y práctica de riesgo para la transmisión del VIH. Las variables clínicas estudiadas incluyen, complicaciones clínicas en relación con la infección VIH al inicio y final del seguimiento así como la mortalidad al final del estudio. Referente al tratamiento; se recogió el tratamiento antirretroviral prescrito, otros tratamientos concomitantes así como datos en relación con la cumplimentación y efectos secundarios derivados de éstos. Asimismo, se llevó a cabo una historia clínica estructurada por órganos y aparatos en la que se recogen datos anamnésicos y semiológicos.

\section{VARIABLES ANALÍTICAS}

Se realizó una analítica básica a todos los pacientes que incluían parámetros hematimétricos, VSG, bioquímica completa así como parámetros inmunológicos y virológicos. El diagnóstico de la infección VIH se realizó mediante ELISA (Enzygnost Anti HIV 1-2 Plus, Behring Diagnostics) y fue confirmado mediante western blot (New lab blot I, Sanofi Diagnostics, Pasteur). Los recuentos de linfocitos CD4 y CD8 se realizaron por citometría de flujo. Para la realización de la carga viral se utilizó la técnica de retrotranscripción-reacción en cadena polimerasa( Amplicor HIV Monitor Test, Roche Diagnostics System) y los resultados se expresaron en copias por $\mathrm{ml}$.

\section{VARIABLES DE LA ESFERA PSICOLÓGICA}

La presencia e intensidad de síntomas depresivos fueron explorados mediante el Beck Depresion Inventory que es un instrumento que ha sido utilizado y evaluado psicométrica- 
mente en una amplia variedad de poblaciones psiquiátricas y normales. Es un cuestionario de 21 items y para su interpretación se ha utilizado la valoración de Conde y Useros $(28,29)$, que categoriza la puntuación obtenida (desde 0 a superior a 25) en intervalos que van desde la ausencia de depresión a la presencia de depresión grave.

La presencia de de ansiedad se exploró mediante el Cuestionario de Ansiedad Estado-Rasgo: State-Trait Anxiety Inventory (STAI) descrito por Levitt en 1967 y adaptado para su uso en España por la Sección de Estudios de TEA Ediciones S.A. La escala E (estado) consta de 20 frases con las que el individuo puede describir como se siente "en un momento en particular", mientras que la escala R (rasgo), también con 20 frases puede mostrar como se siente el sujeto "generalmente". La puntuación de esta escala se realiza en forma de valores absolutos o de percentiles siendo la el percentil 50 el punto de corte a partir del cual el paciente se considera que presenta ansiedad.

\section{VARIABLES RELACIONADAS CON LA SITUACIÓN FUNCIONAL Y LA PERCEPCIÓN DEL ESTADO DE SALUD}

Para la valoración de la situación funcional de los pacientes e utilizaron el Performance Status de Karnofsky, asignado por el facultativo que evalúa al paciente según la escala convencional cuya puntuación oscila entre 0 y 100; y el índice de Katz de Independencia en las Actividades de la Vida Diaria, sobre seis labores ya conocidas: bañarse, vestirse, usar el retrete, moverse, contener los esfínteres y alimentarse cuya puntuación oscila entre 0 y 6 .

Para la valoración del estado de salud tal como es percibido por los propios pacientes, se operativiza como una medición cuantitativa a través del índice proporcionado por el Cuestionario de Salud SF-36. Este Cuestionario de Salud SF36 es la versión española, adaptada transculturalmente por Alonso J y cols. del Departamento de Epidemiología y Salud Pública del Instituto Municipal de Investigación Médica de la Universidad Autónoma de Barcelona, del instrumento SF-36 Health Survey, cuestionario autoadministrado que contiene 36 items que abarcan ocho dimensiones de la salud: Función físi ca: explora distintos aspectos en relación a las limitaciones físicas mayores y menores. Limitaciones de rol por problemas físicos: explora las limitaciones en el trabajo y las actividades usuales de los pacientes que se deban a problemas físicos. Dolor corporal: explora la cantidad de dolor corporal y su interferencia con las actividades normales. Percepción de salud general: explora la percepción de resistencia a la enfermedad y de estado de salud. Vitalidad: explora el nivel de energía y fatiga. Función social: explora la cantidad y calidad de las actividades sociales. Limitaciones de rol por problemas emocionales: explora las mismas limitaciones que el apartado b, pero debido a problemas emocionales. Salud mental: explora de manera global las principales dimensiones de la salud mental. La puntuación de la escala varía de 0 a 100 en cada dimensión siendo 0 el peor estado y 100 el mejor para esa dimensión concreta. Esta escala incluye además un último ítem: Transición de salud referida; que explora si existen cambios percibidos en las dimensiones del estado de salud a lo largo del último año. Una valoración positiva se deduce de un descenso en la puntuación de la escala.

\section{ANÁLISIS ESTADÍSTICO}

Todas las variables recogidas en el protocolo fueron registradas en un soporte informatizado que garantizaba la confidencialidad de los datos. Se realizó inicialmente una estadística descriptiva simple que incluirán medias, desviaciones estándar y medianas para los datos cuantitativos y proporciones para los cualitativos, especificando los intervalos de confianza al 95\%. Se analizaron las diferencias entre grupos para lo que se utilizarán los procedimientos de contraste de hipótesis adecuados (Chi cuadrado, t de Student, test de Wilcoxon o ANOVA).Para evaluar los cambios entre la primera y segunda evaluación se llevará a cabo un análisis pareado mediante la t de Student pareada y el test de Wilcoxon. El análisis multivariante se realiza mediantes un modelo de regresión lineal. Se consideró significación estadística $\mathrm{p}<=0,05$.

\section{RESULTADOS}

\section{CARACTERÍSTICAS GENERALES Y SOCIOECONÓMICAS}

De los 52 pacientes estudiados, $45(86,5 \%)$ eran varones y 7 mujeres. La edad media de la población era de 34 años (SD 8). La mayoría de los pacientes $34(65,4 \%)$ estaban solteros, $11(21,2 \%)$ estaban casados, $4(7,7 \%)$ divorciados y $3(5,8 \%)$ viudos. Un $50 \%$ (26 pacientes), seguían viviendo en domicilio paterno, seguido de $14(26,9 \%)$ que lo hacían con su pareja y 5 $(9,6 \%)$ vivían solos. El resto lo hacía en casas de acogida o alberges . Entre los pacientes que vivían en domicilio propio o familiar, la media de convivientes era de 3,46 (SD 2) con una mediana de 4 (1-11). En cuanto al nivel cultural de la muestra, la mayoría de los pacientes habían cursado estudios primarios hasta $5^{\circ}$ de EGB o poseían el título de graduado escolar; 20 $(38,4 \%)$ y $18(34,6 \%)$ respectivamente, ningún paciente poseía título universitario medio o superior y el resto se repartían entre el analfabetismo, ausencia de estudios o la finalización de bachiller superior. Sólo 9 (17,3\%) pacientes trabajaban activamente. Dieciocho $(34,6 \%)$, se encontraban en situación de desempleo, $11(21,1 \%)$ de baja por enfermedad y el resto se encontraba en jubilación anticipada u otras situaciones. El nivel de ingresos económicos anuales medios por familia era de 1,78 (SD 1,15) millones de pesetas .La práctica de riesgo principal para la transmisión del VIH fue la adicción a drogas por vía parenteral en un 41 pacientes $(78,8 \%)$, seguida de las relaciones heterosexuales de riesgo y homosexuales. Nueve pacientes $(17,3 \%)$ mantenían una adicción activa a drogas inhaladas y ningún paciente mantenía una drogadicción parenteral activa. Diecinueve pacientes $(36,5 \%)$ estaban en programa de deshabituación con metadona. Veintitrés pacientes $(44,2 \%)$ habían estado al menos una vez en prisión.

\section{SITUACIÓN CLÍNICA Y EVOLUTIVA AL COMIENZO DE ESTUDIO}

El tiempo medio de evolución de la población desde el momento del diagnóstico de la infección VIH, hasta el momento de la primera entrevista fue de 69,7 (SD 30,6) meses, de los cuales 17,2 (SD 10) meses lo eran de situación de enfermedad avanzada. Hasta ese momento, 45 pacientes habían desarrollado un total de 74 complicaciones por el VIH durante el período de inmunosupresión avanzada, lo que representa 1.64 (SD 0,5) 
complicaciones por paciente. Esto supone una tasa anual de 1,2 (SD 0,4) complicaciones/año hasta el inicio del estudio. En la figura 1 se representa cómo quedaban categorizados los pacientes al comienzo del estudio (Enero de 1996).

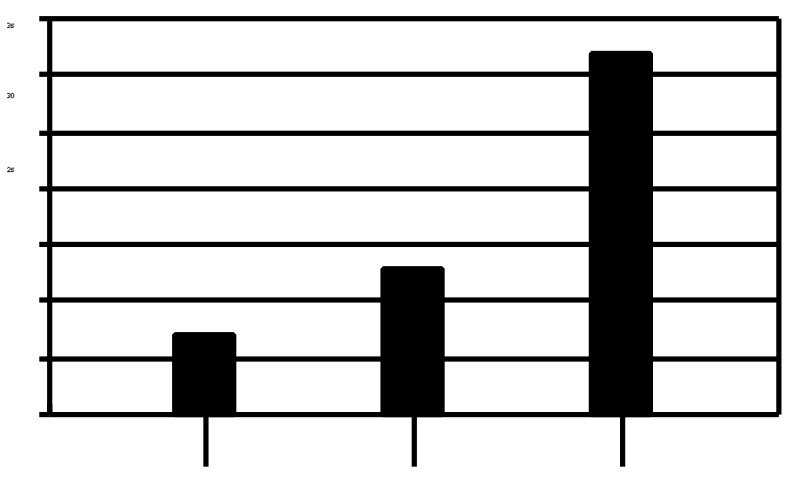

Fig. 1. Clasificación de los pacientes según el CDc-93 al inicio del estudio.

\section{ESTRATEGIAS TERAPÉUTICAS REALIZADAS}

Al inicio del estudio, la mayoría de los pacientes 49 $(94,2 \%)$, se encontraban realizando tratamiento antirretroviral con uno o dos de los fármacos disponibles en nuestro medio hasta ese momento (Tabla I) . La ausencia de tratamiento antirretroviral de tres pacientes al inicio del estudio se debía a la suspensión en los meses previos debido a efectos secundarios y escasa respuesta inmunológica. La duración media de tratamiento antirretroviral hasta ese momento era de 27,6 (SD 18,6) meses. Los cambios de estrategia terapéutica se hicieron siguiendo las recomendaciones internacionales y valorando la situación clínica de los pacientes (Tabla II). En diez pacientes no se produjo ningún cambio de estrategia terapéutica debido a la mala situación clínica o bien por la buena respuesta a los fármacos inicialmente utilizados. El tiempo medio de duración de la nueva terapia antirretroviral en los pacientes en que ésta fue instaurada fue de 6,5 (SD 2,9) meses.

La cumplimentación de las pautas de tratamiento antirretroviral prescrita fue correcta en el 84,3\% de los casos, sin que se encontraran diferencias significativas entre las diferentes estrategias de tratamiento realizadas a lo largo del estudio $[\mathrm{RR}=1,44$, IC 95\%(0,52-3,97); $\mathrm{p}=0,47]$. Un total de 32 pacientes $(61,2 \%)$ presentaban algún tipo de efectos secundarios debido a la medicación antirretroviral prescrita.

\section{TABLA I}

TRATAM IENTO ANTIRRETRO VIRAL AL INICIO DEL ESTUDIO

\begin{tabular}{lc}
\hline Tratamiento antirretroviral & Número de pacientes \\
\hline AZT & 6 \\
AZT + ddC & 19 \\
AZT + ddl & 21 \\
ddC & 3 \\
Ninguno & 3 \\
\hline
\end{tabular}

\section{TABLA ॥}

TRATAM IENTO ANTIRRETRO VIRAL EN LA SEGUNDA VALORACIÓ N DE LOS PACIENTES

\begin{tabular}{lc}
\hline Tratamiento antirretroviral & Número de pacientes \\
\hline$A Z T+d d C$ & 3 \\
$A Z T+d d l$ & 4 \\
$A Z T+3 T C$ & 2 \\
$3 T C+D 4 T$ & 7 \\
$A Z T+d d I+$ Saquinavir & 2 \\
$A Z T+3 T C+$ Saquinavir & 5 \\
ddI+D4T+Indinavir & 4 \\
ddC+3TC+Saquinavir & 2 \\
$3 T C+D 4 T+$ Saquinavir & 12 \\
$3 T C+D 4 T+$ Indinavir & 6 \\
$3 T C+D 4 T+$ Ritonavir & 2 \\
Ningún tratamiento & 3 \\
\hline
\end{tabular}

\section{EVOLUCIÓN CLÍNICA DE LA POBLACIÓN A LO LARGO DEL ESTUDIO}

Desde que los pacientes fueron entrevistados por primera vez, 26 pacientes tuvieron un total de 40 complicaciones clínicas en relación con la infección VIH. Esto representa una tasa anual de 0,75 (SD 0,7) complicaciones/año, inferior al período avanzado previo al inicio del estudio $(\mathrm{p}=0,05)$. Ocho pacientes fallecieron por estas complicaciones durante el período de estudio lo que representa un 15,3\% de mortalidad de la población en el año de seguimiento.

\section{VALORACIÓN CLÍNICA}

En relación a los hallazgos clínicos principales que son obtenidos de las entrevistas realizadas al inicio y fin del estudio son representados en la tabla III. Los parámetros de laboratorio son representados en la tabla IV. Estos datos traducen una importante prevalencia de síntomas estudiados con una tendencia a la mejoría al final del estudio en cuanto a la reducción significativa y tendencia a disminuir la presencia de síntomas referidos. Del mismo modo se produce un importante beneficio inmunológico con la introducción de las nuevas estrategias de tratamiento antirretroviral incrementándose significativamente los recuentos de linfocitos CD4 y con una importante reducción de la carga viral.

\section{ESTUDIO DE LA DEPRESIÓN}

Al inicio del estudio, solamente 7 pacientes $(13,4 \%)$ estaban libres de depresión según la escala de BECK. El resto de la muestra analizada presentaba algún grado de depresión y en el $25 \%$ de los pacientes (13) ésta alcanzaba rango de gravedad. Cuando se vuelve a repetir este estudio al final del seguimiento se constata una significativa reducción del número de 
TABLA III

SINTO M ATO LO GÍA M ÁS FRECUENTEM ENTE REFERIDA

\begin{tabular}{|c|c|c|c|}
\hline Síntomas referidos & 1' Entrevista & 2a Entrevista & Significación \\
\hline Astenia & $35(67 \%)$ & 27 (SD 64,5) & RR $0,9(0,4-1,9) ; p=0,8$ \\
\hline Anorexia & $25(48 \%)$ & 7 (SD 16,7) & $\operatorname{RR~} 0,6(0,4-0,8) ; p=0,02$ \\
\hline Sudoración & $21(40,1 \%)$ & $8(S D 19,1)$ & $\operatorname{RR~} 0,7(0,5-1,01) p=0,07$ \\
\hline Nauseas & $20(38,4 \%)$ & 7 (SD 16,7) & $\operatorname{RR} 0,7(0,5-1,1) ; p=0,1$ \\
\hline O dinofagia & $14(26,9 \%)$ & $2(\mathrm{SD} 4,8)$ & RR $0,7(0,6-0,96) ; p=0,058$ \\
\hline Tos & $30(57,1 \%)$ & $20(S D 47,6)$ & $\operatorname{RR} 0,79(0,4-1,3) ; p=0,5$ \\
\hline M ialgias & $21(40,3 \%)$ & $13(\mathrm{SD} 30,4)$ & $\operatorname{RR} 0,75(0,4-1,4) ; p=0,5$ \\
\hline Pérdida de peso & $16(30,5 \%)$ & $2(\mathrm{SD} 4,4)$ & $\operatorname{RR} 0,7(0,5-0,9) ; p=0,03$ \\
\hline Cefalea & $18(34,6 \%)$ & 7 (SD 16,7) & $\operatorname{RR~} 0,8(0,6-1,06) ; p=0,18$ \\
\hline $\begin{array}{l}\text { Polisintomáticos } \\
\text { (M ás de } 3 \text { síntomas) }\end{array}$ & $44(84,6 \%)$ & $23(54,8 \%)$ & RR $0,7(0,5-0,95) ; p=0,05$ \\
\hline $\begin{array}{l}\text { Indice M asa Corporal } \\
\left(\mathrm{Kg} / \mathrm{m}^{2}\right)\end{array}$ & $20,1(S D 2,7)$ & 21,6 (SD 3) & $p=0,09$ \\
\hline
\end{tabular}

TABLA IV

DETERM INACIO NES ANALÍTICAS REALIZADAS

\begin{tabular}{|c|c|c|c|}
\hline Parámetros & 1’a Entrevista & 2a Entrevista & Significación \\
\hline Hemoglobina & 12,1 (SD 2) & $12,6($ SD 2,3$)$ & $p=0,5$ \\
\hline Linfocitos totales & 650(SD 528) & 1090(SD 810) & $p=0,05$ \\
\hline Plaquetas & 166575(SD 77133) & $145400(S D 63000)$ & $p=0,07$ \\
\hline Velocidad de sedimentación & $82,5(S D 31)$ & 75,1 (SD 31) & $p=0,1$ \\
\hline Proteínas totales & $8,4(\mathrm{SD} 1,3)$ & $8,3(\mathrm{SD} 1,4)$ & $p=0,3$ \\
\hline Inmunoglobulina igg & 1879,5(SD 731) & $1790,8(S D 807)$ & $p=0,3$ \\
\hline Prealbúmina & 16,9 (SD 8) & $20,2($ SD 10,6$)$ & $p=0,1$ \\
\hline Trigliceridos & 240(SD 144) & 235(SD 147) & $p=0,8$ \\
\hline Got & 64 (SD 44) & $66,3(S D 44)$ & $p=0,7$ \\
\hline Linfocitos cd4 (cel/ $\mu \mathrm{l})$ & 78(SD 68,7) & $250,3(S D 163)$ & $p=0,0001$ \\
\hline Carga viral (copias/ml) & 207917(SD 140000) & 66481(SD 79000) & $p=0,003$ \\
\hline$\beta 2$ microglobulina (mg/l) & $3,2(S D 1,2)$ & $2,8(S D 0,9)$ & $p=0,1$ \\
\hline TSH & $4,9(S D 3,1)$ & $4,2(S D 3,5)$ & $p=0,6$ \\
\hline
\end{tabular}

pacientes que presentaban una depresión moderada o grave 8 $(19,04 \%)$ y $3(7,1 \%)$ respectivamente, frente al $26,9 \%$ y $25 \%$ en la primera revisión; [RR 0,7 $(0,4-0,9) ;$ p=0,05]. Los resultados se representan en la figura 2 .

\section{ESTUDIO DE LA ANSIEDAD}

En la primera valoración de los pacientes, un total de 40 pacientes $(76,9 \%)$ presentaban estado de ansiedad de los que en $30(75 \%)$ ésta presentaba criterios de gravedad. En la segunda entrevista $26(61,9 \%)$, estado de ansiedad de los cuales en la mayoría $22(73,3 \%)$ ésta era de intensidad levemoderada. Esto representa una reducción del número de pacientes que vivían con una respuesta de ansiedad las circunstancias que los rodeaban [RR 0,6 (0,3-1,09); p=0,07] así como de la gravedad de este trastorno [RR 0,1 $(0,04-0,79)$; $\mathrm{p}=0,009]$. El rasgo de ansiedad se mantiene invariable a lo largo del seguimiento lo cual es esperable y sirve de validez interna de la aplicación de la escala. Estos resultados se representan en la figura 3. 


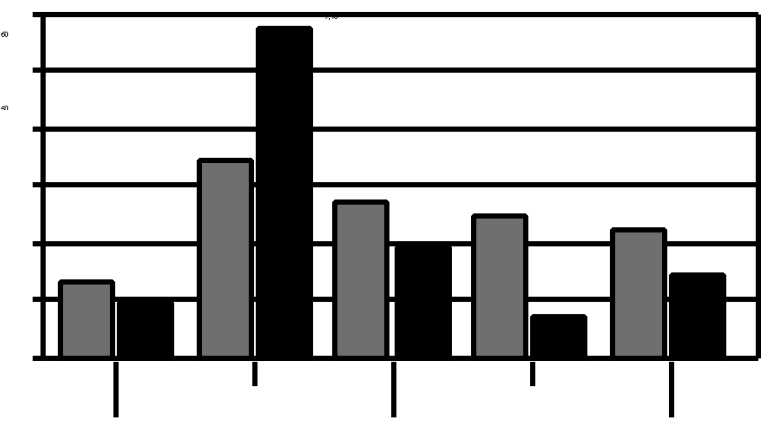

$\square$

Fig. 2. Resultados del estudio de la depresión mediante la aplicación de la escala de Beck.

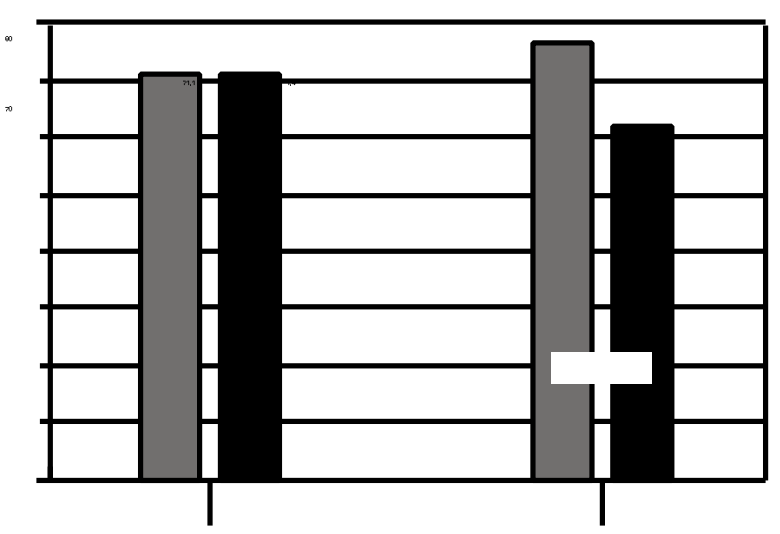

Fig. 3. Resultados del estudio de ansiedad mediante la aplicación del instrumento STAI.

\section{CAPACIDAD FUNCIONAL}

Mediante el Karnofsky Performence Status, se obtuvo en la primera entrevista una puntuación media de la escala de 75 (SD 12) mientras que en la segunda entrevista ésta se incrementó a 85,6 (SD 11); $\mathrm{p}=0,02$, lo que representa una mejoría significativa de la capacidad funcional valorada por el médico a lo largo de este estudio. El índice de Katz de Independencia de las Actividades de la Vida Diaria no mostró alteración a lo largo del seguimiento, obteniéndose en ambas entrevistas, una mediana de puntuación de 6 con rangos de (3-6) y (4-6) respectivamente para la primera y segunda valoración de los pacientes. Esto resultados ponen de manifiesto la ausencia de repercusión de la infección avanzada por el VIH en cuanto a la independencia de la vida diaria en esta población.

\section{PERCEPCIÓN DE LA SALUD PROPIA}

Los resultados de la puntuación obtenida en las 8 dimensiones del cuestionario SF-36, se representa en la figura 4. Se constata una baja puntuación en la primera entrevista en la mayoría de las dimensiones del instrumento SF-36 sólo alcanzando una puntuación aceptable en las dimensiones de función física y social. En la segunda entrevista, se siguen manteniendo resultados aceptables en las funciones física y social, produciéndose además mejorías significativas en la limitación del rol por problemas físicos $(\mathrm{p}=0,02)$ y la percepción de salud general $(\mathrm{p}=0,03)$, a pesar de lo cual aún se seguía presentando una baja puntuación en la escala de esta última dimensión. El resto de las dimensiones, seguían manteniéndose con bajas puntuaciones sin cambios significativos sobre la primera entrevista. Con respecto al cambio de salud percibida por los pacientes, obtenemos una puntuación media en la primera entrevista de 3,2 (SD 1,3) mientras que en la segunda entrevista la puntuación obtenida fue de 2,5 (SD 0,8), lo que representa un descenso significativo de la puntuación media obtenida en la segunda valoración de los pacientes $(\mathrm{p}=0,02)$. Este resultado pone de manifiesto la valoración de una mejoría en el estado de salud percibida por la población a lo largo del estudio.

\section{ANÁLISIS MULTIVARIANTE}

Con objeto de conocer cuales son las variables que se relacionan más significativamente con cambios en aquellas de la esfera psicológica y de calidad de vida analizadas en este estudio, se realizó un análisis multivariante utilizándose múltiples variables recogidas en este estudio que abarcan diversos aspectos de los pacientes tales como clínicos, analíticos, sociales y económicos. Estas variables se analizaron junto a las escalas de los instrumentos empleados para la exploración de la esfera psicológica y calidad de vida.

\section{ESFERA PSICOLÓGICA}

En el modelo multivariante construido $(r=0,82 ; p<0,001)$, las variables que se relacionaban significativamente con la presencia de depresión, ansiedad y con la severidad de estos trastornos fueron; el encontrarse en estadios más avanzados del CDC 1993 ( $\beta=1,1, p=0,0004)$, tener mayor nivel cultural $(\beta=0,97, p=0,002)$, de ingresos económicos $(\beta=0,8, p=0,01)$ y los pacientes con transmisión sexual del VIH $(\beta=0,2, p=0,05)$.

\section{ESTADO DE SALUD PERCIBIDO}

\section{Función física}

En el modelo multivariante construido $(r=0,93 ; p<0,0001)$, el efecto sobre la función física fue negativo en los pacientes en estadios más avanzados del CDC $(\beta=-1,13, p=0,0001)$, con mayor estado de ansiedad $(\beta=-1,1, \mathrm{p}=0,0004)$, mayor nivel cultural $\beta=-1,07, p=0,005)$ y mayores ingresos económicos $(\beta=-0,78, p=0,01)$.

\section{Rol físico}

Al analizar la limitación del rol por problemas físicos, en el análisis multivariante $(r=0,56 ; p=0,007)$, fueron los pacien- 


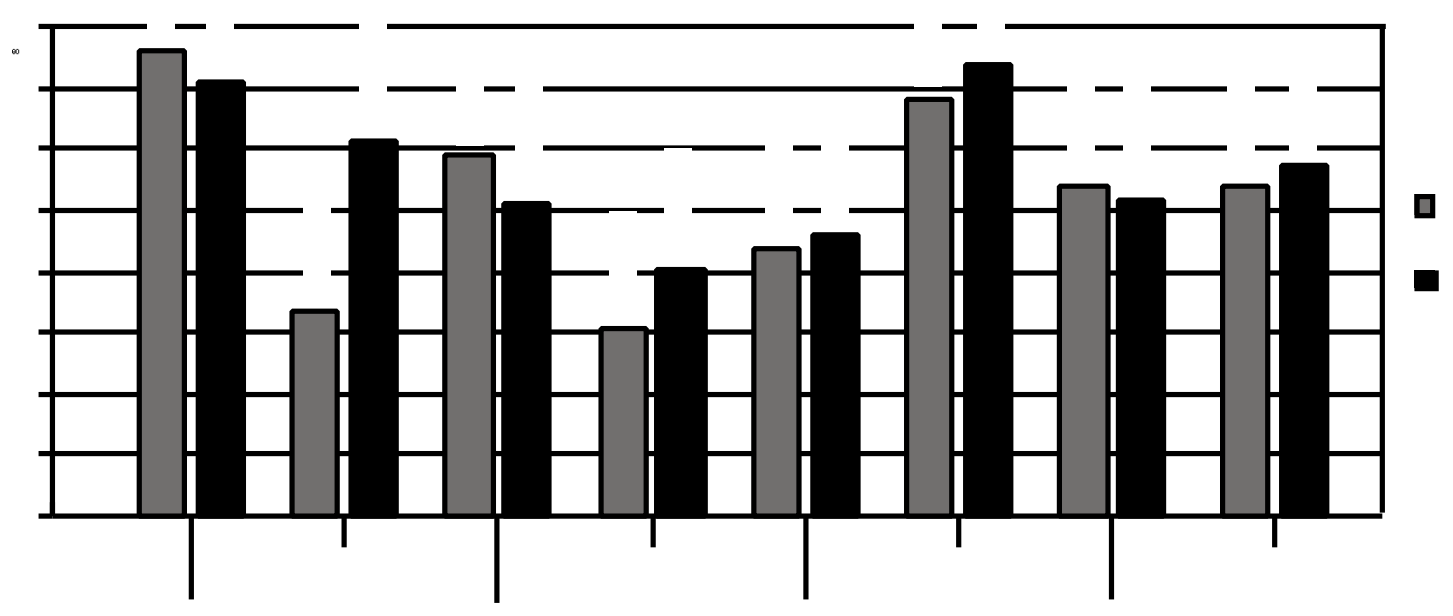

Fig. 4. Estados de salud percibiba según SF-36.

tes con estadios más avanzados del CDC y con mayores ingresos económicos presentaban una menor puntuación en esta dimensión $(\beta=-1,07, p=0,005$ y $\beta=-0,74, p=0,05$ respectivamente).

\section{Dolor corporal}

En relación al dolor corporal referido por el paciente $(\mathrm{r}=0,79, \mathrm{p}=0,001)$, sólo los pacientes con estadios más avanzados del CDC mostraban puntuaciones más bajas en esta dimensión, lo que representa una peor situación en esta categoría $(\beta=-0,74, p=0,05)$.

\section{Salud general}

En relación con los cambios en la percepción general de la salud personal $(\mathrm{r}=0,93, \mathrm{p}=0,0001)$ fueron los pacientes con estadios más avanzados del CDC $(\beta=-0,38, \mathrm{p}=0,06)$ que presentaron más baja puntuación en esta dimensión.

\section{Vitalidad}

En relación a la vitalidad como dimensión, $(r=0,91$, $\mathrm{p}=0,0001)$ fueron los pacientes con estadios más avanzados del CDC $(\beta=-1,02, p=0,0006)$, mayores ingresos económicos ( $\beta=-0,7, p=0,003)$ y menor número de convivientes familiares $(\beta=0,57, p=0,004)$, los que mostraban puntuaciones más bajas en ésta dimensión.

\section{Salud mental}

Ena relación al estado de salud mental percibida $(\mathrm{r}=0,90, \mathrm{p}=0,005)$, los pacientes con estadios más avanzados del CDC $(\beta=-0,93, p=0,01)$, mayor nivel de ingresos económicos $(\beta=-1,33, p=0,01)$, menor número de convivientes familiares $(\beta=0,63, p=0,01)$, fueron los que presentaron más baja puntuación en esta dimensión.

\section{Función social}

En relación a la calidad de la actividad social desarrollada por los pacientes $(\mathrm{r}=0,77, \mathrm{p}=0,002)$, los pacientes con mayor número de convivientes $(\beta=0,64, p=0,03)$, presentaban una menor interferencia en la función social que desarrollaban, sin que ninguna otra variable se relacionara con modificaciones significativas de este aspecto de la calidad de vida.

\section{Rol emocional}

El análisis multivariante pone de manifiesto $(r=0,58$, $\mathrm{p}=0,005)$ que los pacientes con presencia y mayor severidad de depresión $(\beta=-1,1, p=0,01)$, los que convivían con un menor número de familiares $(\beta=0,4, \mathrm{p}=0,06)$ y los que tenían un menor nivel de estudios $(\beta=0,82, p=0,002)$, eran los que más problemas de rol por causa emocional presentaban.

\section{Transición de salud referida por el paciente}

Al realizar el análisis multivariante $(\mathrm{r}=0,75 ; \mathrm{p}=0.001)$ se pone de manifiesto que son los pacientes con estadios más avanzados del CDC $(\beta=-0,94, p=0,02)$, los paciente con ausencia o menor severidad de la depresión $(\beta=1,5, p=0,004)$, los de mayor grado de ansiedad $(\beta=-0,7, \mathrm{p}=0,04)$, los de mayor nivel cultural $(\beta=-1,6, p=0,002)$ y los que convivían con menor número de familiares $(\beta=0.65, \mathrm{p}=0,01)$ son los que percibieron un cambio más positivo en su estado de salud en el período que duró el estudio. 


\section{DISCUSIÓN}

En esta muestra de 52 pacientes con infección avanzada por el VIH, los datos demográficos la sitúan en el contexto de los de la infección VIH de nuestro entorno, con predominio de varones infectados y donde la adicción a drogas por vía parenteral es la práctica de riesgo más prevalente para la transmisión de la infección VIH (30). Son pacientes con un bajo nivel social, cultural y que en su mayoría permanecen solteros y viviendo en domicilio paterno. Al inicio del estudio, los pacientes se encuentran muy sintomáticos siendo también destacable el IMC medio de 20,1 (SD 2,7), que puede considerarse bajo teniendo en cuenta que la población está constituida fundamentalmente por varones. Probablemente existen factores ajenos a la propia infección VIH tales como la adicción activa a drogas inhaladas que pueden estar contribuyendo a algunos de los hallazgos clínicos.

$\mathrm{Al}$ analizar la situación de los pacientes desde el punto de vista de la esfera psicológica, llama la atención la elevada prevalencia de trastornos de ansiedad y depresión, superior a la que cabría esperar en pacientes crónicos y con problemática económica o social, lo cual denota que la infección VIH avanzada tiene un impacto importante sobre este aspecto clínico. Son además los pacientes en estadios más avanzados los que presentan más incidencia y gravedad de este trastorno además de los de mayor nivel sociocultural y los que presentaban un transmisión heterosexual del virus. Es en estos pacientes en los que la situación de deterioro a la que les ha conducido la infección VIH avanzada probablemente más ha cambiado sus vidas, al contrario de los pacientes de menor nivel sociocultural (mayoría de ADVPs) que presentan una mayor incidencia de complicaciones relacionadas con su condición social y práctica de riesgo, por lo que el impacto psicológico de la infección VIH podría haber sido menor por este motivo. Las disfunciones en la esfera psicológica pueden tener repercusiones sobre la propia evolución de la enfermedad mediado por la falta de adherencia a los tratamientos, revisiones o por efecto sobre el propio sistema inmunológico, siendo frecuentemente un aspecto infravalorado por el clínico.

Encontramos al inicio del estudio una importante limitación funcional valorada por el médico y del estado de salud que perciben los pacientes, en la esfera física y psíquica con relativa conservación de la función social. En el análisis multivariante se pone de manifiesto que es el estadiaje de la infección según el CDC 93 (pacientes más avanzados) la variable que más fuertemente condiciona cambios en las diversas dimensiones de salud percibida. Además, los pacientes con mayor nivel sociocultural perciben también un mayor deterioro de la salud sobre todo en la función física, vitalidad y mayor incidencia de problemas laborales. La explicación de este hallazgo puede tener los mismos fundamentos que en el caso de los problemas de salud mental. La incidencia de trastornos ansioso-depresivos es más elevada en estos pacientes con mayor nivel sociocultural, lo que confiere una mayor dificultad en un entorno socio-laboral probablemente con más responsabilidad. El deterioro de la salud mental de los pacientes también condiciona un deterioro de la calidad de vida de éstos; se percibe un deterioro de la función física y problemática socio-laboral derivada del deterioro emocional. Sin embargo, ninguna de estas variables relacionadas con la salud mental tienen repercusión en el análisis multivariante sobre el deterioro psíquico percibido por la población. Este resultado en principio sorprendente puede poner de manifiesto la falta de reproductibilidad del instrumento SF-36 a la hora de detectar verdaderos cambios en el estado de salud mental percibida teniendo en cuenta que se trata de una población con una gran prevalencia de síntomas físicos y psíquicos en la que puede ser difícil detectar cambios en la salud mental percibida por los pacientes separadamente del importante deterioro físico sufrido por éstos.Es de destacar el efecto positivo de la convivencia familiar en relación a un menor deterioro de la salud percibida en diversas dimensiones.

Al contrastar los datos obtenidos referentes a la calidad de vida de estos pacientes con los de estudios llevados a cabo con el mismo instrumento (SF-36) en muestras pertenecientes a poblaciones de pacientes con patologías crónicas, como son la EPOC, hipertensión arterial, diabetes, insuficiencia cardiaca o la depresión, se constata una peor situación de la salud percibida por los pacientes VIH avanzados (30-34).

En la evolución de los pacientes tras la introducción de las nuevas estrategias de tratamiento antirretroviral se constata un claro beneficio clínico global para los pacientes. El tamaño de la muestra y la duración del período de estudio ha impedido unos resultados aún más contundentes. La mejoría de la situación física de los pacientes se acompaña paralelamente de una clara mejoría de la su situación de salud mental, con un significativo descenso en el número de pacientes con trastornos más severos de depresión y ansiedad. También mejora la capacidad funcional de los pacientes percibida tanto por el clínico como por los propios enfermos. Son precisamente los pacientes con estadios más avanzados del CDC y con más carga de ansiedad al inicio del estudio, los que perciben un mayor beneficio. Sin embargo, los pacientes con mayor gravedad de síntomas depresivos son los que menos cambios en la salud perciben. Estos resultados traducen el hecho de que los pacientes con enfermedad VIH más avanzada y con más ansiedad generada, son los que más ganan en salud percibida al mejorar inmunológicamente. Sin embargo, la depresión, una vez establecida tiene un importante efecto en la calidad de vida de la población y puede alterar la percepción de mejorías del estado de salud por la alteración del estado de ánimo. Por todo ello es importante considerar este trastorno dentro del enfoque terapéutico de la infección VIH avanzada.

Por tanto, la infección VIH avanzada conlleva una importante carga sintomática que causa un considerable deterioro de la salud percibida tanto por el clínico y por el propio paciente y ésta afecta a dimensiones físicas, psíquicas y sociales, comprometiéndose de manera importante la calidad de vida de la población. Estos pacientes se benefician de la introducción de los nuevas estrategias de tratamiento antirretroviral(35). La mejoría inmunológica se acompaña de una mejor situación física y psíquica percibida por el clínico y por los pacientes. Sin embargo existen aspectos de ésta como es el caso del deterioro de la salud mental a la que probablemente habrá que prestar más atención dentro de un enfoque más globalizador de este tipo de pacientes en aras de conseguir una mejor calidad de vida de la población afectada. 


\section{Bibliografía}

1. Oddone EZ, Cowper P, Hamilton JD, et al. Cost effectivnes analysis of early zidovudine treatment of HIV infected patients. BMJ 1993; 20: 1322-5.

2. Wu AW, Rubin HR, Mathews WC, Brysk LM et al. Functional status and well being in placebo controlled trial of zidovudine in early symptomatic HIV infection. J Acquir Immune Defic Syndr 1993; 6: 452-8.

3. Gelber RD, Lenderking WR, Cotton DJ, et al. Quality of life evaluation in a clinical trial of zidovudine therapy in patients with mildy symptomatic HIV infection. The AIDS Clinical Trial Group. Ann Intern Med 1992; 116: 961-66.

4. Testa M, Simonson D. Assesement of quality of life outcomes. N Engl J Med 1996; 334: 835-40.

5. Clearly P, Wilson I, Fowler F. A theoretycal framework for assesing and analyzing helath related quelity of life. Av Med Sociol 1994; 5: 23-41.

6. Wetchel T, Piette J, Mor V, et al. Quality of life in person with HIV infection: Measurment by the Medical Outcome Study Instrument. An Intern Med 1992; 116: 129-67.

7. García Huete E. SIDA. Apoyos del entorno personal, social y laboral. Madrid: Eudema, 1993.

8. Organización Mundial de la Salud: Atención Primaria de Salud. Informe de la Conferencia Internacional sobre Atención Primaria de Salud Alma-Ata. Ginebra, World Health Organization, 1978.

9. Stevens JC, Poston JW, Walker SR: Measuring the quality of life. Proc Guild Hosp Pharmacists 1986; 22: 39-49.

10. Tsevat J, Solzan JG, et al. Health values of Patients Infected with Human Immunodeficiency Virus. Relationship to Mental health and Phisical Functioning. Med Care 1996; 34: 44-57.

11. Lubeck DP, Fries JF. Assesement of quality of life in early stage of HIV-infected persons. Data from the AIDS Time-oriented Health Outcome Study (ATHOS). Qual Life Res 1997;6:494-506.

12. Lutgendorf S, Antoni MH, Schneiderman N, Fletcher MA. Psichological counseling to improve quality of life in HIV infection. Patient Educ Couns 1994; 24: 217-35.

13. Ganz PA, Coscarelli Schag CA, Petersen L, Hirji K. Describin the health related quality of life impact of HIV infection: finfings from a study using the HIV Overview of Problems-Evaluation System (HOPES). Qual Life Res 1993; 2; 109-119.

14. Lubeck DP, Fries JF. Changes in the quality of life among persons with HIV infection. Qual Life Res 1992; 6: 359-66.

15. Paul D, Cleary PhD et al. Health related Quality of Life in Persons with Acquired Immune Deficiency Syndrome.Med Care 1993; 31: 569-80.

16. Holmes WC, Bix B, Meritz M, et al. Human immnunodeficinecy virus (HIV) infection and quality of life: The potential impact of Axis I psychiattric disorders in a sample of 95 HIV seropositive men. Psichosom Med 1997; 59: 187-92.

17. Clearly PD, Fowler FJ, et al. Health related quality of life in personst with acquired immune defficiency syndrome. Med Care 1993; 31: 56980
18. Leibrich P, Engeter M, Olbrich E, Rubbert A, et al. Longitudinal development od distress, coping and quality of life in HIV-positive persons. Psychoter Psychosom 1997; 66: 237-47.

19. Wachtel T, Piete J, Mor V, Stein M, et al. Quality of life in persons with human immunodediciency virus infection:measurement by the Medical Outcome Study Instrument. Ann Intern Med 1992; 116: 129-37.

20. Niyongabo T, Bouchaud O, et al. Nutritional status of HIV seropositive subjects in an AIDS clinic in Paris. Eur J Clin Nutr 1997; 51: 637-40.

21. Von Roenn JH. Management of HIV-related bodyweight loss. Drugs 1994; 47: 774-89

22. Carretero MD, Burgess AP, et al. Reliability and validity of an HIVspecific health related quality of life measure for the use with injecting drug users. AIDS 1996;10: 1699-705.

23. Sowell RL, Seals BF, et al. Quality of life in HIV-infected woment in the south-eastern United States. AIDS Care 1997; 9: 501-12

24. Aguirrebengoa K, Mallolas J, Uriz J, Torres Tortosa M. Análogos nucleósidos inhibidores de la transcriptasa inversa: Utilidad en pacientes adultos infectados por el VIH. Enf Infecc Microbiol Clin 1996; 14 (Supl. 1): 3-9.

25. Rubio R, Romeu J, Viciana $\mathrm{P}$, Redondo C. Inhibidores de la proteasa en el VIH. Enf Infec Microbiol Clin 1996; (Supl. 1): 10-4

26. Consejo Asesor Clínico del Plan Nacional sobre el SIDA. Tratamiento antirretroviral del adulto. Ministerio de Sanidad y Consumo. Noviembre de 1996.

27. Monés Xiol J , Barrio Medrano Jl. Aspectos éticos de la práctica médica en el SIDA. Ann Med Interna (Madrid) 2000; 17: 171-3.

28. Conde V y Useros E. Adaptación castellana de la escala de evaluación conductual para la depresión de Beck. Revista de Psiquiatría y Psicología Medica de América y Europa 1975; 12: 217-36.

29. Conde V, Esteban P y Useros E. Revisión crítica de la adaptación castellana del cuestionario de Beck. Revista de Psicología General Aplicada 1976; 31: 469-97.

30. Marco Martínez J, Barba Martín R et al. Cambio en el perfil de los pacientes VIH positivos. ¿Que ha pasado en los últimos cinco años? Ann Med Interna (Madrid) 2000; 17: 174-7.

31. Nerenz DR, Repasky DP, Withehouse FW and Kahkonen DM. Ongoing assessement of health status in patients with diabetes mellitus. Med Care 1993; 30 (supl.): MS112-MS124.

32. Wells KB, Burnam MA, Rogers W, Camp P. The course of depression in adult outpatients. Results from the medical Outcome Study. Arch Gen Psych 1992; 49: 788-94.

33. Tarlor AR, Ware JE, Greenfield S et al. The Medical Outcome Study: An application of methods for monitoring the results of medical care JAMA 1989; 262: 925-930.

34. Stewart AL and Ware JE (Eds). Measuring functioning and well being The Medical Outcomes Study aproach. Durham NC: Duke University Press 1992.

35. E. Pujol de la Llave. Tratamiento Antirretroviral en 1999:de la incertidumbre a la esperanza. Ann Med Interna (Madrid) 1999; 16: 215-7. 